\title{
New Method to Restore Fading Papanicolaou Specimen Using an Optical Clearing Agentx
}

Kiyotada Washiya $^{1 *}$, Makoto Sakurai ${ }^{1}$, Saki Furutate ${ }^{1}$, Estuko Okusawa ${ }^{2}$, Shigeharu Hatakeyama ${ }^{3}$, Haruhiko Yoshioka ${ }^{1}$ and Jun Watanabe ${ }^{1}$

${ }^{1}$ Department of Pathologic Analysis, Hirosaki University Graduate School of Health Sciences, Japan

${ }^{2}$ Department of Pathology, Hachinohe City Hospital, Hachinohe, Japan

${ }^{3}$ Cytology \& Pathology Research Center, Bunkyo-ku, Tokyo 113-0034, Japan

\begin{abstract}
Cytological specimens are used only once for staining, and cannot be reused after preservation in paraffin blocks. If a technique is developed allowing restaining of precious/rare specimens or those for education, which have been discolored due to long-term preservation, to restore their former states, it will be extremely useful for the reuse of specimens. We performed this study to determine whether discolored specimens can be restored to their former condition by applying an optical clearing agent.
\end{abstract}

For restaining, 131 Papanicolaou (Pap) specimens prepared from 13 to more than 30 years ago were used. Using an optical clearing agent (SCALEVIEW-A2), Pap restaining, endurance tests of the optical clearing agent by repeated use, and immunostaining were performed.

After treatment with the optical clearing agent for5-10 minutes, discolored cytological specimens could be stably restained just as the initial Pap specimens. As a result of the immunostaining of specimens treated using this method, both the nucleus (Ki-67) and cell membrane (MOC31) were positively stained. The optical clearing agent did not deteriorate when used repeatedly ten times.

Discolored precious cytological specimens could be restored using a optical clearing agent. Specimens processed using this method could also be immunostained, and so this method is extremely useful.

Keywords: Fading papanicolaou specimen; Optical clearing agent; Re-stain

\section{Introduction}

Unlike histological specimens that can be prepared any time by cutting paraffin blocks into a desired number of thin sections, cytological specimens are used only once. Therefore, discolored specimens due to long-term preservation cannot be reused. However, if a technique is developed enabling the restaining of precious and/or rare specimens or those for education to restore their former state, it may be extremely useful for the reuse of specimens. Techniques such as decolorization with $1 \%$ hydrochloric acid followed by restaining of Papanicolaou (Pap) specimens, the addition of 33\% glycerine [1] or a surfactant to the Pap stain [2], or treatment with phosphate buffer (reported only in domestic society meetings) have been used. However, the reproducibility of restoration to the state before discoloration was low, and there have been only a few published papers.

We have studied methods to restore discolored specimens due to long-term preservation to favorably stained specimens. In this study, favorable specimens could be prepared using an optical clearing agent [3] that was originally developed for the highly detailed observation of deep areas such as nerves and capillaries of the organ by multiphoton laser scanning microscopy.

\section{Materials and Methods}

\section{Samples}

A total of 95 discolored cytological specimens (56 uterine cervix, 29 endometrial, and 10 sputum specimens) prepared from 13 to more than 30 years ago were used (Table 1). In addition, for immunostaining with antibodies Ki-67 (MIB-1, Dako) and MOC31 (monoclonal anti-MOC-31, Dako), a total of 33 specimens (11 uterine cervix, 11 gastrointestinal, 3 mammary gland, 2 pleural effusion, and 6 ascites specimens) were used (Table 2).

\section{Methods}

Detachment of cover glasses: (1) Specimens were placed in xylene, and cover glasses were removed (heating at $37^{\circ} \mathrm{C}$ or $60^{\circ} \mathrm{C}$ overnight when necessary).

(2) The mounting agent was removed using new xylene ( 1 hour $\mathrm{x} 3$ times)

\begin{tabular}{|c|c|}
\hline Material & Specimen \\
\hline Cervix & 56 \\
\hline Endometium & 29 \\
\hline Sputum & 10 \\
\hline
\end{tabular}

Table 1: Pap specimens for restaining.

\begin{tabular}{|c|c|}
\hline Material & Specimen \\
\hline Cervix & 11 \\
\hline Digestive organ & 11 \\
\hline Breast & 3 \\
\hline Peural effusion & 2 \\
\hline Ascites & 6 \\
\hline
\end{tabular}

Table 2: Specimens for immunostaining.

*Corresponding author: Kiyotada Washiya, Department of Pathologic Analysis, Hirosaki University Graduate School of Health Sciences, 66-1 Honcho, Hirosaki-shi, Aomori 036-8564, Japan, Tel. +81 17239 5972; E-mail: kwashiya@cc.hirosaki-u.ac.jp

Received October 23, 2013; Accepted February 17, 2014; Published February 19, 2014

Citation: Washiya K, Sakurai M, Furutate S, Okusawa E, Hatakeyama S (2014) New Method to Restore Fading Papanicolaou Specimen Using an Optical Clearing Agent. J Cytol Histol 5: 219. doi:10.4172/2157-7099.1000219

Copyright: ( 204 Washiya K, et al. This is an open-access article distributed under the terms of the Creative Commons Attribution License, which permits unrestricted use, distribution, and reproduction in any medium, provided the original author and source are credited. 
(3) Xylene was removed with $100 \%$ ethanol (15 minutes $\mathrm{x}$ twice)

(4) Specimens were passed through a descending ethanol series (95-70\%) for 5 minutes each, and washed with water for 2 minutes.

Decolorization of old Pap specimens: Both our new method and 2 control methods were used.

1. Control method 1: Placement in 1\% HCL ethanol for 15 minutes (without use of buffer)

2. Control method 2: Placement in 1\% HCL in ethanol for 15 minutes, followed by in phosphate buffer $(\mathrm{pH}, 7.2)$ for 1 hour

3. Our new method: Clearing with an optical clearing agent (SCALEVIEW-A2), which was developed by the RIKEN Science Institute [3] and is sold by Olympus, followed by placement in phosphate buffer $(\mathrm{pH}, 7.2)$ to terminate the reaction of the clearing agent. The optical clearing agent was composed of $4 \mathrm{M}$ urea, $10 \%$ glycerol, and $0.1 \%$ Triton X-100.

\section{Time of treatment with the optical clearing agent}

Specimens were immersed in the optical clearing agent for 3, 5, 10, 20,30 , or 60 minutes, and the appropriate treatment time was evaluated.

Pap restaining: Basically, the routine method was used. However, the time for differentiation in $0.5 \%$ HCL in $70 \%$ ethanol after staining with hematoxylin was slightly longer (30-40 dips) than in the routine method.

Immunostaining: Immunostaining was performed to determine whether discolored specimens can be immunostained. After the cover glasses of old Pap specimens were removed, they were decolorized with $1 \% \mathrm{HCl}$ ethanol and an optical clearing agent, and immunostained with Ki67 (1:50 dilution), which stains nuclei, and MOC31 (1:200 dilution), which stains cell membranes, using a Leica automated immunostainer (Bond-MAX). Antigen retrieval was performed by microwave heating for 10 minutes in $0.01 \mathrm{M}$ citrate buffer $(\mathrm{pH}, 6.0)$ for Ki67 and in $0.01 \mathrm{M}$ citrate buffer $(\mathrm{pH}, 6.0)$ on Bond-MAX for 40 minutes for MOC31.To prepare discolored cytological specimens under the same conditions, multiple cell transfer specimens were prepared from a single specimen according to the quick cell transfer technique [4] described by Itoh et al. with modification, and comparison and evaluation were made. The cell transfer technique is shown in Table 3.

Endurance tests of the optical clearing agent: When multiple specimens are treated with an optical clearing agent, the effects of the agent may deteriorate. Therefore, to determine whether the effects of a clearing agent deteriorate or persist when many specimens are treated with the same clearing agent without replacement, $50 \mathrm{ml}$ of a clearing agent was placed in a round dish and repeatedly used. Five specimens were treated each time, which was repeated 10 times (a total of 50 specimens). The specimens used consisted of 40 endocervical carcinoma and 10 gastrointestinal adenocarcinoma specimens (Table 1).

\section{Results}

\section{Comparison of Pap restaining (Figure 1a-h)}

Figure 1a: Control method 1: The nuclei were generally lightly stained without sharpness.

Figure 1b: Control method 2: Some nuclei were intensely stained, but others were lightly stained, showing inconsistent stainability. There was neither sharpness nor transparency
Figure 1c: Treatment with an optical clearing agent for 3 minutes: The nuclei were sharply stained, and the contrast was clear. Compared with treatment with a clearing agent for 5 or 10 minutes, the nuclei were intensely stained, and the degree of transparency was low. However, reproducibility was high compared with the control methods.

Figure 1d: Treatment with an optical clearing agent for 5 minutes: Nuclear staining was sharp, and contrast was clear. The nuclei were transparent, and reproducibility was high for re-straining.

Figure 1e: Treatment with an optical clearing agent for 10 minutes: This treatment as well as treatment for 5 minutes resulted in sharp nuclear staining and high contrast. The nuclei were transparent.

Figure 1f: Treatment with an optical clearing agent for 20 minutes: Slight nuclear swelling was observed, and the degrees of transparency and sharpness decreased compared with treatment for 10 minutes.

Figure 1g: Treatment with an optical clearing agent for 30 minutes: Compared with treatment for 20 minutes, artifacts (swelling) were further added, the nuclei were lightly stained, and nuclear chromatin granules were difficult to identify.

Figure 1h: Treatment with an optical clearing agent for 60 minutes: Compared with treatment for 30 minutes, neutrophils in the background were swollen, and nuclear stainability in epithelial cells was poor.

Comparison of keratinizing-type squamous cell carcinoma cells showed differences in the intensity of nuclear and cytoplasmic stains and obscure nuclear margins after Control method 2, but intense nuclear and cytoplasmic stains with high reproducibility after treatment with the clearing agent for 10 minutes. The optimal treatment time with a clearing agent was 5-10 minutes.

Specimens preserved for a long period varied in their condition; some were almost completely discolored, and others showed preservation of only nuclear stainability. However, restaining after treatment using this method resulted in high and stable stainability (Figure 2).

\section{Immunostaining with Ki-67 or MOC31}

(1) Ki-67 (Figures 3a and 3b)

Figure 3a: Treatment with an optical clearing agent: Ki-67 was positive in the nuclei of all specimens, and repeated use of the optical clearing agent (10 times) did not decrease immunological stainability. The nuclei of not only solitary cells but also 3-dimensional epithelial cell clusters were intensely stained dark brown.

Figure 3b: Control method 2: Scattered solitary cells were stained. However, in 3-dimensional cell clusters, marginal cells, not the whole

\section{Cell transfer technique}

(1) Dilute Malinol with an equal volume of xylene.

(2) Cover the glass slide after detaching the cover slip with $1 \mathrm{ml}$ of diluted Malinol.

(3) Place Malinol solidified on an $80^{\circ} \mathrm{C}$ hot plate for $30 \mathrm{~min}$

(4) Dip the solidified thin membrane into $60^{\circ} \mathrm{C}$ water for $15 \mathrm{~min}$ to soften it.

(5) Peel off the softened Malinol membrane with forceps.

(6) Trim the membrane into pieces.

(7) Place the trimmed pieces on new silane-coated glass slides in warm water.

(8) Remove excess air bubbles and water using tissue paper.

(9) Dry glass slides on an $80^{\circ} \mathrm{C}$ hot plate for $30 \mathrm{~min}$. 


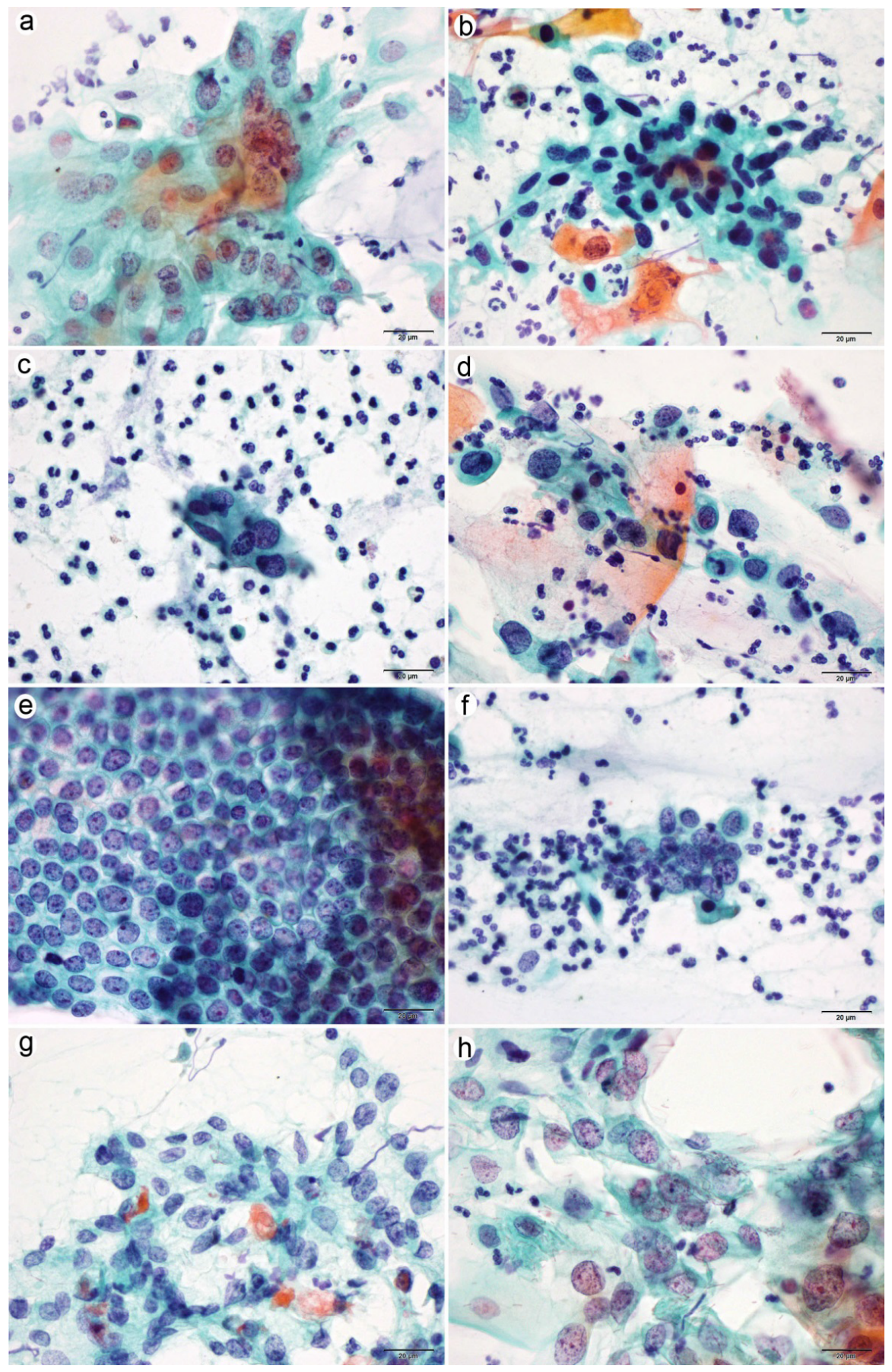

Figure 1: Comparison of Pap restaining methods.
a. Control 1
b. Control 2
c. Optical clearing agent, $3 \mathrm{~min}$
d. Optical clearing agent, $5 \mathrm{~min}$
e. Optical clearing agent, $10 \mathrm{~min}$
f. Optical clearing agent, $20 \mathrm{~min}$
g. Optical clearing agent, $30 \mathrm{~min}$
h. Optical clearing agent, $30 \mathrm{~min}$
i. Optical clearing agent, $60 \mathrm{~min}$. 


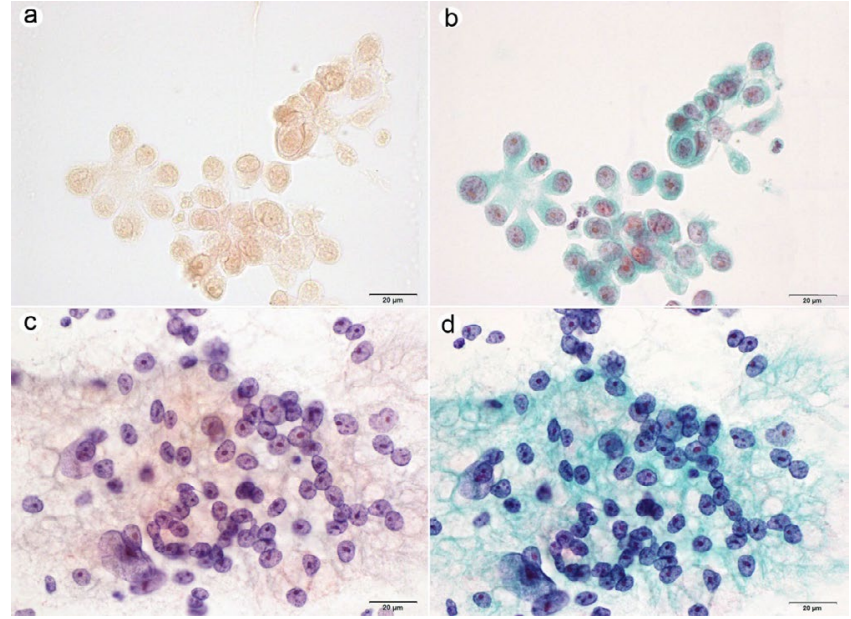

Figure 2: Comparison between discolored and restained specimens. a. Discolored specimens(sputum)

b. Pap-restained specimens (Optical clearing agent, $10 \mathrm{~min}$ )(sputum)

c. Discolored specimens(uterine cervix)

d. Pap-restained specimens (Optical clearing agent, $10 \mathrm{~min}$ ) (uterine cervix).

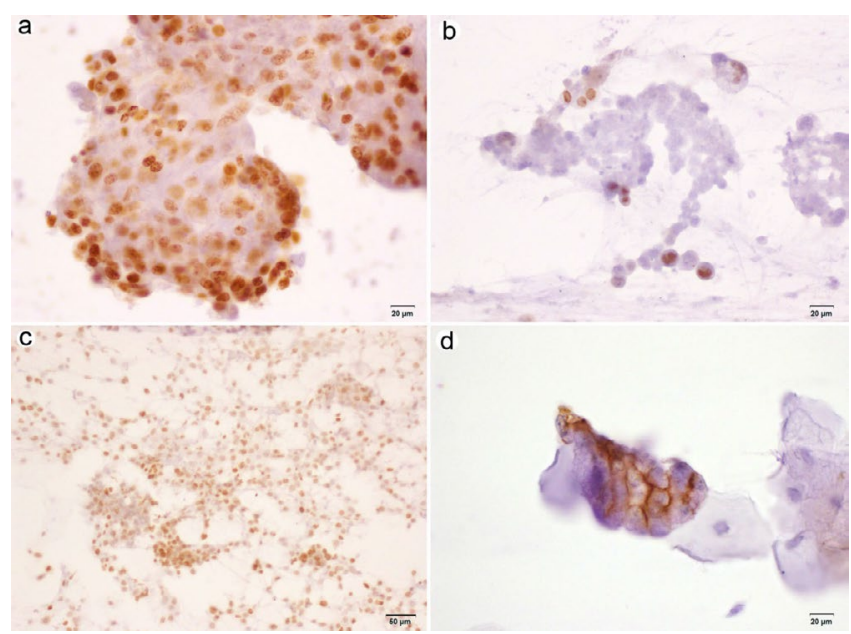

Figure 3: Immunocytochemical staining after treatment with an optical clearing agent.

a. Ki-67 Clearing agent, $10 \mathrm{~min}$ (pleural effusion)

b. Ki-67 Control 2(pleural effusion)

c. MOC31 Clearing agent, $10 \mathrm{~min}$ (ascites)

d. MOC31 Control 2 (ascites).

area of the masses, were lightly stained (positive image). The number of positive cells was smaller than that after treatment with a clearing agent.

\section{(2) MOC31 (Figures $3 c$ and d)}

Figure 3c: Method with an optical clearing agent: There were many positive cells with the cell membrane stained dark brown. Repeated use of the clearing agent did not decrease stainability.

Figure 3d: Control 2: After Control method 2, MOC31 was also positive in many cells.

Thus, treatment with a clearing agent enabled immunostaining of old Pap specimens. Although some specimens could not be immunologically stained, we speculated that problems with the initial Pap specimen preparation and the conditions of specimen preservation unfavorably affected these specimens compared with others positive for the same antibody.

\section{Influences of repeated use of the optical clearing agent}

The effects of Pap restaining persisted even after repeated use (10 times) of the clearing agent, suggesting good cost-performance. In addition, the effects of immunostaining (Ki-67, MOC31) were also persistent.

\section{Discussion}

Optical clearing agents [3] were developed for the highly detailed observation of deep areas of the body such as the nerves and capillaries using a multiphoton laser scanning microscope (FV1200MPE) [5] without damaging specimen tissue (awarded a 2012 Edison Gold Award). We used this reagent to revitalize Pap specimens discolored due to long-term preservation. Specimens restained after treatment using this method can be readily observed employing a conventional light microscope, not a special multiphoton laser scanning microscope. As a result of Pap restaining after treatment with an optical clearing agent, transparency was achieved after 5-10 minutes, and cells and nuclei were sharply stained, showing high stainability. Cytological specimens preserved for a long period varied in their condition; both the cytoplasm and nucleus were almost completely discolored in some specimens, and only nuclear stainability remained in others (Figure 2). However, restaining for 5-10 minutes after treatment using this method resulted in good and stable stainability without nuclear overstaining in all specimens (Figure 2). On the other hand, with an increase in the time of treatment with an optical clearing agent, cells were swollen, and the nuclei were lightly stained. These changes may be due to Triton $\mathrm{X}-100$ as a surfactant contained in the clearing agent, and therefore, the treatment time is important.

In addition, whether immunostaining of old, discolored specimens is possible was determined. Ki-67, which strongly stains the nucleus of carcinoma cells, and MOC31, which strongly stains the cell membrane of epithelial cells, were used as antibodies. Staining with Ki-67 revealed a higher number of nuclear-positive cells and higher staining intensity using treatment with an optical clearing agent than using Control method 2. Staining with MOC31 revealed intensely stained cell membranes using treatment with an optical clearing agent as well as Control method 2. Reactivity may differ among antibody types, but an optical clearing agent increased immunostaining reactivity of old Pap specimens. Further studies for confirmation are necessary. There were some specimens unstained with these antibodies. However, many specimens were positively stained with the same antibodies. We speculate that problems with the initial Pap specimen preparation (cell degeneration, fixation, dehydration, etc.) and the conditions of preservation unfavorably affected these specimens.

Endurance tests of the optical clearance agent showed no deterioration of Pap stains even when the clearing agent placed in a dish was repeatedly used 10 times. The repeated use of the clearing agent also did not affect stainability with Ki-67 or MOC31. We were concerned about the possible deterioration of the effects of the clearing agent due to the mixture of water and impurities in the process of treating many specimens. However, the effects of the optical clearing agent did not decrease even after it was used 10 times without replacing it. Further repeated use may be possible, and the cost-performance of this agent is excellent.

As major causes of discoloration, changes in the chemical structure due to photoreactions and dye elution into the mounting agent have 
Citation: Washiya K, Sakurai M, Furutate S, Okusawa E, Hatakeyama S (2014) New Method to Restore Fading Papanicolaou Specimen Using an Optical Clearing Agent. J Cytol Histol 5: 219. doi:10.4172/2157-7099.1000219

Page 5 of 5

Detachment of cover glasses

1. The cover glass is removed by immersion in xylene (heating at $37^{\circ} \mathrm{C}$ or $60^{\circ} \mathrm{C}$ overnight when necessary).

\begin{tabular}{|l|l|}
\hline 2. The mounting agent is removed using new xylene. & $1 \mathrm{hr} \times 3$ times
\end{tabular}

\begin{tabular}{l|l}
\hline $3.100 \%$ ethanol (removal of xylene) & $15 \mathrm{~min} \times 2$ times
\end{tabular}

4. Specimens are passed through a descending ethanol series (95-70\%) for 5 minutes each, and washed with water (2 min).

Decolorization of old Pap specimens

Specimens are treated with an optical clearing agent (SCALEVIEW-A2) for 5-10 minutes and placed in phosphate buffer (pH, 7.2) to terminate the reaction of the clearing agent.

Pap staining

Basically, the routine staining method is used. However, it is recommended to use a longer time (30-40 dips) for differentiation after hematoxylin staining.

Table 4: Washiya method of Papanicolaourestaining.

been suggested. Dye elution into the mounting agent may occur due to dye elution into water and ethanol remaining after inadequate dehydration or clearing, or due to the influences of the components of the mounting agent themselves or impurities [For example, Canada balsam contains organic acids such as abietic acid $(\mathrm{C} 19 \mathrm{H} 29 \mathrm{COOH})]$. Discoloration is slower in specimens with a large amount of cells and in a low-temperature, low-humidity environment. Among the 3 dyes of Pap staining, light green is more soluble in water than eosin or orange $\mathrm{G}$, and its discoloration tends to be rapid. In previous studies, methods such as the addition of $33 \%$ glycerine [1] or a surfactant (Emulgen 810) [2] to the stain solution were used, but stainability was unstable among discolored specimens. Therefore, the present method is very useful. Concerning sputum specimens, when the cover glass was removed by immersion in xylene, cells sometimes detached from the glass [2]. Similar cell detachment occurred using this method. Its cause may be as follows. Since sputum specimens are frequently thick smears, the smear surface develops cracks due to long-term preservation, and sputum has abundant mucus containing glycoproteins and lipids, which is mixed with foreign materials such as house dust, pollen, soot and smoke, bacteria, and viruses, pus mostly consisting of dead white blood cells, and blood, resulting in cell detachment. Evaluation during the removal of cover glasses is necessary. When this method is used, since optical clearing agents sometimes corrode metals such as natural iron, copper, and aluminum, metal equipment, if used, should be adequately washed after use.
Restaining after 5-10 minutes treatment using an optical clearing agent resulted in high and stable stainability. Finally, this method for the restaining of discolored old Pap specimens (Washiya method of Papanicolaou restaining) is summarized in Table 4. We intend to perform further studies to improve not only discolored, but also routine Pap staining specimen by increasing the transparency.

\section{Acknowledgement}

This study was supported by a grant for Hirosaki University Institutional Research (2013).

\section{References}

1. Gill GW (1974) Staining, Compendium on Cytopreparatory Techniques. Chicago.

2. Fukunaga Y, Yashiro S (1979) A New Method of Restaining Old, Discolored Specimens. J JpnSocClinCytol 474-481.

3. Hama H, Kurokawa H, Kawano H, Ando R, Shimogori T, et al. (2011) Scale: a chemical approach for fluorescence imaging and reconstruction of transparent mouse brain. Nat Neurosci 14: 1481-1488.

4. Itoh H, Miyamjima $\mathrm{Y}$, Osamura $\mathrm{Y}$, Tsutomu $\mathrm{Y}$ (2002) A study of quick "cell transfer" technique. J JpnSocClinCytol 41: 302-303.

5. http://download.messe-muenchen.de/media_pub/mediacenter/evdb2/event downloads/analytica2012/21148643/1334819673.pdf 